\title{
Adaptação e Validação da Nova Escala Geral de Autoeficácia
}

\author{
Laércio André Gassen Balsan ${ }^{1}$ (D) \\ Universidade Federal de Santa Maria, Santa Maria-RS, Brasil \\ Laila Leite Carneiro (D), Antonio Virgílio Bittencourt Bastos (D) \\ Universidade Federal da Bahia, Salvador-BA, Brasil \\ Vânia Medianeira Flores Costa (D) \\ Universidade Federal de Santa Maria, Santa Maria-RS, Brasil
}

\section{RESUMO}

Autoeficácia é a crença da pessoa na sua própria capacidade para organizar e executar ações, influenciando experiências humanas positivas. É essencial haver instrumentos capazes de mensurar adequadamente esse construto. Esta pesquisa teve como objetivo adaptar e validar para o contexto brasileiro a New General Self-Efficacy Scale (NGSE), por meio de dois estudos independentes de corte transversal. Em ambos os estudos, a amostra foi composta por adultos de 18 a 66 anos, com variados graus de escolaridade, que responderam a escala após a tradução e adaptação dos itens do instrumento original. A melhor solução encontrada por meio das análises fatoriais exploratórias e confirmatórias apontou para a manutenção de seis dos oito itens originais da escala em uma estrutura unifatorial. O modelo final apresentou índices de ajuste satisfatórios $\left(\chi^{2} / g l=2,98 ; \mathrm{GFI}=0,97\right.$; $\mathrm{CFI}=0,97$; TLI=0,94; RMSEA=0,08), assim como níveis adequados de consistência interna $(\alpha=0,83)$, indicando que a medida pode ser utilizada em novas pesquisas nacionais.

Palavras-chave: autoeficácia; escala; validação.

\section{ABSTRACT - Adaptation and validation of the New General Self-Efficacy Scale}

Self-efficacy is a person's belief in their own ability to organize and perform actions, influencing positive human experiences. It is essential to have instruments capable of adequately measuring this construct. This study aimed to adapt and validate the New General Self-Efficacy Scale (NGSE) for the Brazilian context, through two independent cross-sectional studies. In both studies, the sample consisted of adults from 18 to 66 years of age, with varying levels of education, who responded to the scale after the translation and adaptation of the original instrument items. The best solution found through exploratory and confirmatory factor analysis indicated the maintenance of 6 of the 8 original scale items in a single-factor structure. The final model presented satisfactory fit indices $\left(\chi^{2} / g l=2.98 ; \mathrm{GFI}=0.97\right.$; $\mathrm{CFI}=0.97$; TLI $\left.=0.94 ; \mathrm{RMSEA}=0.08\right)$, as well as adequate levels of internal consistency $(\alpha=0.83)$, indicating that this measure can be used in new national studies.

Keywords: self-efficacy; scale; validation.

\section{RESUMEN - Adaptación y Validación de la Nueva Escala General de Autoeficacia}

La autoeficacia es la creencia en la propia capacidad de organizar y ejecutar acciones, influyendo así, en las experiencias humanas positivas. Es fundamental contar con instrumentos capaces de medir este constructo de manera adecuada. Esta investigación tuvo como objetivo adaptar y validar la New General Self-Efficacy Scale (NGSE) para el contexto brasileño, a través de dos estudios transversales independientes. En ambos estudios, la muestra estuvo constituida por adultos de 18 a 66 años, con diferentes niveles de educación, que respondieron a la escala después de la traducción y adaptación de los ítems del instrumento original. La mejor solución encontrada, a través del análisis factorial exploratorio y confirmatorio, señaló la necesidad de corrección de 6 de los 8 ítems de la escala original en una estructura unifactorial. El modelo final presentó índices de ajuste satisfactorios $\left(\chi^{2} / g l=2.98 ; \mathrm{GFI}=0.97\right.$; $\mathrm{CFI}=0.97 ; \mathrm{TLI}=0.94 ; \mathrm{RMSEA}=0.08)$, así como niveles adecuados de consistencia interna $(\alpha=0.83)$, lo que indica que esta medida puede ser utilizada en nuevas investigaciones nacionales.

Palabras clave: autoeficacia; escala; validación.

O construto de autoeficácia foi proposto por Bandura em 1977 com a publicação da obra Self-efficacy: Toward a unifying theory of behavior change (Bandura, 1977). A autoeficácia é um dos principais componentes da teoria social cognitiva (Araújo \& Moura, 2011; Azzi \& Polydoro, 2006; Bandura, 1977; Lent, Brown, \& Hackett, 1994; Olaz, 2003; Pajares \& Olaz, 2008), a qual explica o comportamento humano mediante um modelo da 
reciprocidade triádica (comportamento humano, fatores pessoais e fatores ambientais) (Pajares \& Olaz, 2008).

Autoeficácia foi definida por Bandura (1977) como a crença que as pessoas desenvolvem sobre as suas capacidades pessoais para iniciar, executar e desempenhar com sucesso suas tarefas, as quais exigem esforço e perseverança face às adversidades. Assim, o conceito está ligado à possibilidade de modificação do comportamento por meio de uma mudança na percepção do indivíduo em relação a sua capacidade e aos meios pelos quais as mudanças podem ocorrer. Ou seja, é uma crença do sujeito em sua capacidade de produzir efeitos desejados por meio de suas próprias ações.

Segundo Bandura, Taylor, Williams, Mefford e Barchas (1985, p. 15), "uma teoria que nega que os pensamentos podem regular as ações não dá a explicação do complexo comportamento humano". Segundo Bandura (2008), a perspectiva da agência se contrapõe aos princípios behavioristas que baseavam os processos de aprendizagem na associação entre os estímulos ambientais e as respostas individuais. $\mathrm{Na}$ concepção da agência, o indivíduo influencia o ambiente e contribui para as circunstâncias de sua vida, não sendo apenas produtos dessas condições. Wood e Bandura, (1989), Bandura (1982, 2001, 2002), Schwarzer (1992), Bandura e Locke (2003) e Zimmerman (2000) explicam que quanto mais forte for a percepção de autoeficácia mais vigoroso e persistente será o esforço do indivíduo; sendo um sistema importante para que este atinja os objetivos e execute com sucesso as tarefas a que se propôs.

Indivíduos com uma percepção mais elevada de autoeficácia têm uma maior capacidade para controlar acontecimentos estressantes e uma maior determinação no momento de resolver essas situações, independentemente do tipo de problemática (Bzuneck \& Guimarães, 2003; Schwarzer \& Schmitz, 2004). Os indivíduos tendem a não se resignar em face de uma experiência de fracasso. As adversidades são simples acontecimentos em um processo de realização de um objetivo (Bandura, 1982, 1977).

Por outro lado, indivíduos que se julgam incapazes de lidar com as exigências da situação evitam envolver-se nela com as mais variadas justificações. Indivíduos com baixa autoeficácia tendem a aceitar as limitações à medida que elas aparecem. Para Schwarzer e Schmitz (2004), a autoeficácia tem uma função motivacional, afetando o comportamento de acordo com a percepção que as pessoas têm de sua capacidade.

Um elevado sentimento de eficácia está relacionado com uma melhor saúde geral, uma maior realização pessoal e uma melhor integração social (Bandura, 1977; Lubbers, Loughlin, \& Zweig, 2005; Scholz, Doña, Sud, \& Schwarzer, 2002; Schwarzer, 1992), com um melhor desempenho profissional (Barros \& Batista-Dos-Santos, 2010; Gist, 1987; Latham \& Pinder, 2005; Lubbers et al., 2005; Rhee, Hur \& Kim, 2017; Stajkovic \& Luthans, 1998), maior satisfação (Canrinus, Helms-Lorenz, Beijaard,
Buitink, \& Hofman, 2012; Caprara, Barbaranelli, Steca, \& Malone, 2006; Judge, Bono, Erez, \& Locke, 2005; Lee \& Oh, 2015; Perdue, Reardon, \& Peterson, 2007; Reizer \& Hetssroni, 2015), uma motivação superior (Chen, Gully, Whiteman, \& Kilcullen, 2000; Ciani, Summers, \& Easter, 2008; Lloyd, Bond, \& Flaxman, 2017; Pajares, 1997; Salas \& Cannon-Bowers, 2001; Schepers et al., 2005), com a diminuição do burnout (Fida, Laschinger, \& Leiter, 2016; Smeds et al., 2019), maior engajamento (Perera, Granziera, \& Mcllveen, 2018), maior comprometimento organizacional (Bzuneck \& Guimarães, 2003; Kusumaninggati, Mukhtar, \& Sujanto, 2018; Lee \& Kim, 2019; Tsai, Tsai, \& Wang, 2011; Yousaf \& Sanders, 2012) e ocupacional (Chan, Lau, Nie, Lim, \& Hogan, 2008; Rots, Aelterman, Vlerick, \& Vermeulen, 2007). Assim, é essencial haver instrumentos capazes de mensurar bem a autoeficácia para uma melhor predição desses e de outros comportamentos humanos.

Atualmente, é possível encontrar, tanto na literatura nacional quanto na literatura internacional, diferentes instrumentos que se propõem a medir o fenômeno "autoeficácia", apoiados em concepções teóricas diversificadas. Segundo Snyder e Lopez (2009), existem duas perspectivas teóricas que orientam a mensuração da autoeficácia: a situacional e a baseada em traços.

A perspectiva situacional reflete as crenças do indivíduo sobre sua capacidade e habilidade para atingir determinados objetivos em circunstâncias e domínios específicos. De acordo com essa abordagem, seria possível apenas, a construção de instrumentos de avaliação específicos para determinados domínios, como, por exemplo, a Escala de Autoeficácia para Orientação Vocacional (Snyder \& Lopez, 2009), a Escala de Autoeficácia para a Amamentação (Oriá \& Ximenes, 2010), a Escala de Fontes de Autoeficácia de Docentes (Iaochite \& Azzi, 2012), Escala de Autoeficácia para o Estabelecimento de Bons Relacionamentos com Famílias no Contexto Neonatal e Pediátrico Hospitalar (Cruz, Angelo, \& Santos, 2017) e Escala de Autoeficácia no Trabalho (Cardoso \& Baptista, 2019).

Já na concepção baseada em traços, os fenômenos psicológicos são considerados duradouros. Sherer et al. (1982) afirmam que experiências de autoeficácia pessoal podem se generalizar para ações que transcendam qualquer situação. Desse modo, segundo Araújo e Moura (2011), muito embora alguns autores considerem autoeficácia como um construto de domínio específico, outros tantos pesquisadores têm considerado a existência de uma autoeficácia mais geral, a qual tem encontrado sustentação empírica por meio das escalas que apresentam adequadas propriedades psicométricas (Chen, Gully, \& Eden, 2001; Judge, Erez, \& Bono, 1998; Sherer et al., 1982; Scholz et al., 2002; Schwarzer, 1992; Schwarzer \& Jerusalem, 1995; Schwarzer \& Hallum, 2008; Schwarzer \& Schmitz, 2004).

Segundo Luszczynska, Scholz e Schwarzer (2005) e Scherbaum, Cohen-Charash e Kern (2006), a autoeficácia 
geral é uma percepção de eficácia pessoal independente da situação. Uma das justificativas dos autores que defendem a medida de autoeficácia geral, seria o benefício que ela teria, descartando a necessidade de criação de um instrumento novo para cada circunstância nova investigada, ampliando assim as possibilidades de comparação de resultados.

A autoeficácia geral, segundo Araújo e Moura (2011), contrasta com a formulação original de Bandura, que conceitualiza a autoeficácia como dependente das tarefas específicas que o indivíduo tem que desempenhar. Contudo, os autores reforçam que o número de estudos sobre a autoeficácia geral tem aumentado. Tais estudos trazem evidências de que a autoeficácia geral se correlaciona com a autoeficácia específica a contextos, além de se demonstrar distinta de outros construtos de autoavaliação.

Para Chen et al. (2001), apesar das formulações diferirem quanto à globalidade do construto, a autoeficácia geral e a específica são coincidentes em outros aspectos. Especificamente, na experiência vicariante e na quantidade de esforço que o indivíduo utilizará na concretização de um determinado objetivo e constituem um determinante fundamental para a adaptação a novos acontecimentos.

Em relação a esse construto mais global, foram desenvolvidas algumas medidas de avaliação, dentre elas, em 1979, Schwarzer e Jerusalem desenvolveram um instrumento para lidar com a adversidade em situações cotidianas e amplas. A primeira versão foi desenvolvida no idioma alemão, contendo 20 itens. Em 1981, a escala foi reduzida para dez itens e, posteriormente, foi sendo traduzida e adaptada para 30 idiomas (Sbicigo, Teixeira, Dias, \& Dell'aglio, 2012).

No Brasil, Souza e Souza (2004) adaptaram a escala original de Schwarzer e Jerusalem (1995) e atribuíram-lhe o nome de Escala de Autoeficácia Geral Percebida (EAGP). Os autores encontraram boa consistência interna $(\alpha=0,81)$; entretanto, somente foram fornecidos dados de fidedignidade.

Sbicigo et al. (2012) analisaram as propriedades psicométricas da Escala em adolescentes do Estado do Rio Grande do Sul, Brasil. Participaram 1.007 estudantes (60,5\% meninas) com idades entre 12 e 18 anos, de escolas públicas. Conforme esperado, resultados de análises fatoriais exploratórias e confirmatórias, realizadas com subconjuntos independentes da amostra total, indicaram a unidimensionalidade da medida. A fidedignidade do instrumento nessa amostra, avaliada pelo alfa de Cronbach, foi de 0,85, sendo considerada alta. Da mesma forma, todos os itens apresentaram correlações item-restante satisfatórias. Os resultados indicam que a versão brasileira da EAGP apresenta evidências de validade e de confiabilidade para avaliar a autoeficácia geral em adolescentes.

Sherer et al. (1982), também desenvolveram um instrumento para a avaliação da autoeficácia geral. O estudo envolveu 376 estudantes e 150 pacientes de tratamento de alcoolismo e considerou como resultados as evidências de validade e fidedignidade para fatores de autoeficácia geral e autoeficácia social. Além de ter sido uma das primeiras escalas de autoeficácia geral a ser desenvolvida, ela é altamente usada em contextos clínicos, educacionais e organizacionais (Chen et al., 2001). A escala contém 17 itens dentro de uma estrutura unifatorial (Sherer \& Adams, 1983). Contudo, segundo Chen et al. (2001), pesquisas posteriores encontraram soluções multifatoriais.

Em 1983, Sherer e Adams desenvolveram um instrumento de medida de autoeficácia geral que enfrenta relevantes críticas na literatura internacional em virtude da presença de construtos correlatos em seus itens e pela divergência na concepção do conceito conforme a Teoria Social Cognitiva. Chen et al. (2001) avaliaram esse instrumento e constataram que a medida possui baixa validade discriminante e de conteúdo dificultando a interpretação dos resultados.

Chen et al. (2001) propuseram o instrumento New General Self-Efficacy Scale (NGSE). Para os autores, a autoeficácia geral, além de influenciar a autoeficácia específica de situações contextuais, é mais resistente a influências passageiras e que, apesar de existirem diferenças, ambas as crenças seriam originadas das mesmas fontes. Após desenvolverem sua medida, a compararam com a medida de Sherer et al. (1982) e verificaram que, mesmo tendo um tamanho menor, a escala demonstrou maior confiabilidade, possibilitando a predição de autoeficácia para uma variedade de tarefas em diversos contextos.

A NSGE, em sua estrutura original, é uma escala unidimensional, composta por oito itens, que apresenta consistência interna adequada e demonstra estabilidade ao longo do tempo. No estudo realizado por seus proponentes (Chen et al., 2001) para a validação, a confiança da escala foi medida por dois surveys administrados para os mesmos sujeitos com um intervalo de duas semanas. A escala apresentou alta consistência interna no Tempo 1 $(\alpha=0,85)$ e Tempo $2(\alpha=0,86)$. Além disso, os coeficientes de confiabilidade do teste reteste ao longo do tempo foram altos: $\mathrm{r}_{\mathrm{t} 1-12}=0,65, \mathrm{r}_{\mathrm{t} 2-\mathrm{t} 3}=0,66, \mathrm{r}_{\mathrm{t} 1-\mathrm{t} 3}=0,62$ (Chen et al., 2001).

Apesar dos altos indicadores de qualidade psicométrica da NGSE (Chen et al., 2001) no exterior, uma busca ampla na literatura científica em bases de dados indexadas demonstrou não haver nenhum estudo de validação desse instrumento voltado para a realidade brasileira, a despeito de sua larga tradução e utilização a nível nacional. Com base nisso, a presente pesquisa tem como objetivo adaptar e validar para o contexto brasileiro a NGSE. Mais especificamente, em uma primeira investigação, buscou-se avaliar as características psicométricas da escala, sua validade e estrutura fatorial para o contexto brasileiro. Considerando a extensão do território nacional e sua diversidade cultural, num segundo estudo, procurou-se 
analisar a validade da escala estudando seu padrão fatorial e estabilidade em outra amostra com características distintas da do primeiro estudo.

Optou-se pela NGSE, pois num estudo realizado por Scherbaum et al. (2006) comparando as principais escalas de autoeficácia geral - Sherer et al. (1982), Schwarzer e Jerusalem (1995) e Chen et al. (2001) -, verificou-se que as três demonstraram propriedades psicométricas aceitáveis, contudo a medida de Chen et al. (2001) foi a que apresentou as propriedades mais desejáveis, bem como vantagens em termos de discriminação de itens, conteúdo dos itens e eficiência relativa da função de informação de teste, ou seja, a capacidade de medir um traço latente de forma mais fiável. Somado a isso, ao invés de se criar um novo instrumento, adaptar um previamente validado em outra língua minimiza custos, tempo e possibilita a comparação de resultados em estudos multicêntricos (Guillemin, Bombardier, \& Beaton, 1993).

Para atingir o objetivo de adaptar e validar para o contexto brasileiro a NGSE, originalmente proposta por Chen et al. (2001), foram realizados dois estudos independentes. No primeiro, foi desenvolvida a etapa de adaptação, que incluiu a tradução e o ajuste dos itens para a população brasileira, assim como o estudo piloto, e foram levantados os primeiros dados psicométricos para validação. No segundo estudo, ocorreu apenas a etapa de validação, que angariou evidências adicionais de validade e confiabilidade da escala.

Esses dois estudos, ambos de natureza quantitativa e de corte transversal, são apresentados aqui separadamente em suas características metodológicas e resultados. Posteriormente, os achados dos Estudos 1 e 2 são discutidos em conjunto.

\section{Estudo 1}

\section{Método}

O Estudo 1 foi dividido em duas etapas a fim de realizar o processo de adaptação e validação da escala NGSE para o Brasil.

$\mathrm{Na}$ etapa de adaptação, o primeiro passo empreendido nesta pesquisa foi a tradução da escala para o português por tradutor especializado. $\mathrm{Na}$ sequência, os itens foram analisados por três especialistas, os quais verificaram a adequação da tradução face ao objetivo de avaliação que se pretendia atingir. Revisada a questão semântica e de conteúdo, bem como a clareza, a objetividade e a precisão da linguagem, o conteúdo dos itens traduzidos e adaptados culturalmente para a realidade brasileira passou por um estudo piloto (pré-teste) realizado com 25 indivíduos. Com base em Malhotra (2019), essa etapa foi encerrada por não serem mais necessárias mudanças no instrumento.

Uma vez completada a etapa de adaptação, seguiu-se para a segunda etapa do Estudo 1: a validação da escala, composta por sua aplicação e pela análise de seus indicadores psicométricos. Os indivíduos que participaram do pré-teste não foram incluídos na amostra de validação.

\section{Participantes}

No Estudo 1, a versão pré-testada foi aplicada a uma amostra composta por 504 indivíduos, residentes em dois estados brasileiros (um do Sul e outro do Nordeste brasileiro). O tipo de amostragem foi não probabilístico por conveniência, amostragem característica em estudos envolvendo pessoas, os quais nem sempre permitem a viabilidade de amostras aleatórias. Ou seja, participaram da pesquisa indivíduos que, ao serem convidados, aceitaram colaborar e assegurou-se o anonimato e a privacidade, resguardando-lhes o direito, inclusive, de não responderem ao questionário, se assim o desejassem.

Com idades entre 18 e 59 anos (média $=32,47$ ), a maioria da amostra foi composta por mulheres $(67,7 \%)$. Do total de respondentes, a metade é solteira $(49,7 \%)$. Há um predomínio de católicos $(63,1 \%)$ e, com relação à escolaridade, a grande maioria ao menos terminou o ensino médio (29,1\%). O nível instrucional dos participantes variou de ensino fundamental $(4,6 \%)$ até doutorado $(4,2 \%)$.

\section{Instrumento}

A escala adaptada para o Brasil foi aplicada junto a um questionário para levantamento de dados demográficos. São exemplos de itens da escala: "Eu vou ser capaz de atingir a maioria das metas que eu estabeleci para mim mesmo"; "Eu acredito que posso ter sucesso, sem muito esforço, nas metas que estabeleço para mim mesmo"; "Mesmo quando as coisas estão difíceis, eu consigo desempenhos muito bons". Na mensuração dessa medida foi usada uma escala do tipo Likert, de cinco pontos, em que a atribuição do número 1 representa "Discordo totalmente", e o número 5, "Concordo totalmente".

\section{Procedimentos de Coleta de Dados}

A coleta de dados se deu por meio da aplicação de um questionário estruturado. Nessa etapa, o instrumento de pesquisa foi aplicado diretamente em versão impressa aos indivíduos. Para aplicação dos questionários, foi realizado um treinamento com os pesquisadores que fariam sua aplicação, para que a coleta de dados transcorresse da melhor forma possível.

Juntamente com o questionário, os sujeitos da pesquisa receberam o Termo de Consentimento Livre e Esclarecido (TCLE), no qual constavam informações sobre a importância acadêmica da pesquisa, bem como sobre a garantia de anonimato e o caráter voluntário do preenchimento do instrumento, assim como a possibilidade de abandonar a pesquisa, caso o participante desejasse, atendendo, assim, às recomendações do Comitê Nacional de Ética na Pesquisa (CONEP). O TCLE preenchido e assinado pelo participante. 


\section{Procedimentos de Análise dos Dados}

A análise dos dados foi viabilizada por meio dos softwares SPSS 21.0 (Software Package for Social Sciences) e AMOS. Primeiramente, foram realizadas análises estatísticas exploratórias para identificar e corrigir problemas de digitação, dados omissos (missings) e casos extremos (outliers). As avaliações subsequentes pelos testes de skewness, kurtosis e do teste Kolmogorov-Smirnov demonstraram adequação da amostra em termos de normalidade. Em seguida, conduziu-se as análises fatoriais exploratórias (AFE) e as análises fatoriais confirmatórias (AFC) da escala.

Realizou-se uma análise fatorial exploratória, por meio do método de extração de Análises de Componentes Principais (ACP) (conforme sugestão de Field, 2009) e rotação oblíqua (direct oblimin), considerando previamente como critérios o KMO igual ou superior a 0,60 e o Teste de Esfericidade de Bartlett (qui-quadrado, $\left.\chi^{2}\right)$ significativo $(p<0,05)$ (Tabachnick $\&$ Fidell, 2019). Ressalta-se que a retenção de fatores se baseou também em uma avaliação que considerou tanto a disposição do screenplot quanto o critério de autovalor (eigenvalue) maior que 1,0. Visando encontrar uma solução que correspondesse melhor ao construto, em termos teóricos e psicométricos, foram utilizados dois critérios: (a) foram excluídos itens que não apresentaram carga fatorial em nenhum dos fatores ou valor de comunalidade muito abaixo dos demais itens do fator; e (b) foram excluídos itens que se acoplaram a fatores para os quais não havia sustentação teórica.

Para a AFC, utilizou-se o programa AMOS com o objetivo de testar hipóteses específicas sobre a estrutura latente do modelo, apresentando os respectivos indicadores "goodness" ( $\chi^{2}$ (qui-quadrado), Goodness-of-Fit index (GFI), Adjusted Goodness-of-Fit index (AGFi), RootMean-square Error of Approximation (RMSEA), Comparative Fit index (CFI), Tucker-Lewis Index (TLI), Normed Fit Index (NFI)), os quais permitem avaliar a qualidade de ajuste do modelo a que se propõe (Hair, Black, Babin, Anderson, \& Tatham, 2009).

\section{Resultados}

Ao realizar a AFE da Escala Geral de Autoeficácia, os itens 1 (Eu vou ser capaz de atingir a maioria das metas que eu estabeleci para mim mesmo) e 4 (Eu acredito que posso ter sucesso, sem muito esforço, nas metas que estabeleço para mim mesmo), apresentaram baixa comunalidade: 0,013 e 0,060 , respectivamente. Assim esses itens foram excluídos. Como observa-se na Tabela 1 , o fator único que emergiu após as exclusões citadas apresentou valor próprio de 2,96, explicando 49,37\% da variância total.

Tabela 1

AFE da Escala Geral de Autoeficácia - Estudo 1

\begin{tabular}{|c|c|c|}
\hline \multirow{2}{*}{ Itens } & \multicolumn{2}{|c|}{ Componente } \\
\hline & F1 & $h^{2}$ \\
\hline 8. Mesmo quando as coisas estão difíceis, eu consigo desempenhos muito bons. & 0,753 & 0,557 \\
\hline 5. Eu vou ser capaz de superar com êxito muitos desafios. & 0,749 & 0,561 \\
\hline 6. Eu acredito que posso realizar de forma eficaz muitas tarefas diferentes. & 0,736 & 0,541 \\
\hline 3. Em geral, eu acho que posso obter os resultados que são importantes para mim. & 0,696 & 0,485 \\
\hline 2. Quando me deparo com tarefas difíceis, tenho certeza de que vou realizá-las. & 0,650 & 0,423 \\
\hline 7. Em comparação com outras pessoas, eu posso fazer a maioria das tarefas muito bem. & 0,621 & 0,386 \\
\hline Valor próprio & \multicolumn{2}{|c|}{2,96} \\
\hline Número de itens & \multicolumn{2}{|c|}{6} \\
\hline \% da variância explicada & \multicolumn{2}{|c|}{49,37} \\
\hline Alfa de Cronbach & \multicolumn{2}{|c|}{0,785} \\
\hline
\end{tabular}

Legenda. F1=Autoeficácia; $h^{2}=$ Comunalidade. Fonte. Dados da Pesquisa

Considerando os resultados da AFE, realizou-se uma AFC visando testar a adequação da escala. O modelo inicial sugeriu índices de melhorias e assim foi reespecificado com a inclusão da correlação entre os erros e2 (Mesmo quando as coisas estão difíceis, eu consigo desempenhos muito bons) e e6 (Em comparação com outras pessoas, eu posso fazer a maioria das tarefas muito bem) e entre os erros e4 (Em geral, eu acho que posso obter os resultados que são importantes para mim) e e5 (Quando me deparo com tarefas difíceis, tenho certeza de que vou realizá-las).

Assim, verificou-se que os indicadores de qualidade de ajuste foram: $\chi^{2} / g l=2,63$, GFI $=0,983$, CFI $=0,977$, $\mathrm{TLI}=0,951, \mathrm{NFI}=0,969, \mathrm{RMSEA}=0,074(0,00-0,08) \mathrm{e}$ confiabilidade composta 0,772 . Estes se demonstraram adequados, e os resultados da estrutura do modelo estão sumarizados na Figura 1. 


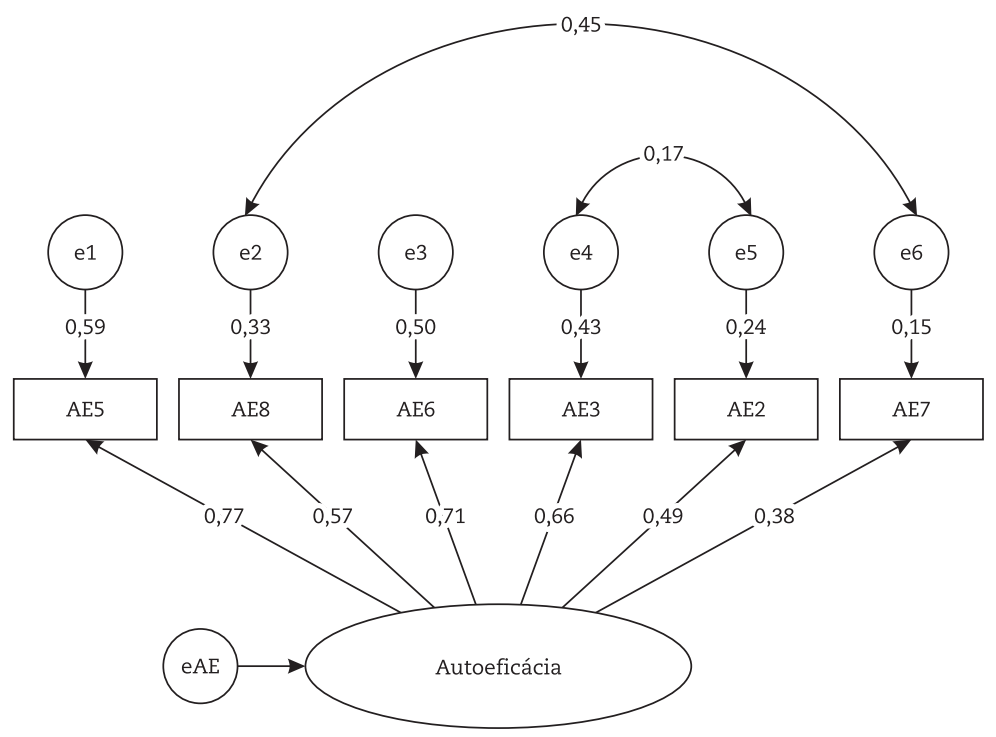

Figura 1. Estrutura do modelo da Escala Geral de Autoeficácia a partir dos dados do estudo 1

Fonte. Dados da pesquisa

Quanto à análise fatorial confirmatória, os resultados apoiam a coerência do modelo teórico que pressupõe sua unifatorialidade. Somado a isso os índices de ajuste observados atendem ao que tem sido considerado apropriado na literatura (Byrne, 2016; Hair et al., 2009; Tabachnick \& Fidell, 2019).

\section{Estudo 2}

\section{Método}

No Estudo 2, foi realizada uma nova validação da escala NGSE para o Brasil, independente do Estudo 1.

\section{Participantes}

Participaram do estudo 398 indivíduos residentes em diversas estados brasileiros (Bahia, Distrito Federal, Espírito Santo, Goiás, Minas Gerais, Rio de Janeiro, Rio Grande do Sul, Paraíba, Pernambuco, Piauí, São Paulo, Santa Catarina, Sergipe). Desses, 21,1\% declararam ocupar cargo de chefia. A maior parte da amostra foi composta por mulheres $(70,1 \%)$, altamente escolarizada $(50,8 \%$ com algum nível de pós-graduação: especialização, mestrado ou doutorado). Houve um equilíbrio em relação ao estado civil dos participantes $(49,7 \%$ de solteiros(as) e $44,2 \%$ de casados(as) ou afins). Em termos de idade, a faixa etária variou de 19 a 66 anos, apresentando como média 35,65 anos $(D P=10,48)$.

\section{Instrumento}

O instrumento foi composto por uma ficha de levantamento de dados demográficos e pela escala NSGE que foi adaptada para o Brasil no Estudo 1. Os oito itens da escala foram avaliados pelos participantes a partir de uma escala de concordância do tipo Likert, de cinco pontos, variando de 1 (Discordo totalmente) a 5 (Concordo totalmente).

\section{Procedimentos de Coleta de Dados}

A coleta de dados foi realizada 14 meses após a coleta realizada para o primeiro estudo. Para facilitar o acesso à quantidade e à variedade de participantes, os instrumentos foram disponibilizados para autoaplicação nas versões impressa (100 respondentes) e digital (298 respondentes) por meio da plataforma SurveyMonkey, para aqueles indivíduos que se dispuseram a participar voluntariamente da pesquisa. Foi utilizada uma amostragem por conveniência, por meio do mecanismo de "bola de neve", disparado por convites para responder a pesquisa para pessoas participantes da rede social dos pesquisadores, sendo solicitado àqueles que recebiam o convite que repassassem para outras pessoas que poderiam estar aptas a responder.

\section{Procedimentos de Análise de Dados}

Para a condução da análise de dados, foram seguidos os mesmos procedimentos descritos no Estudo 1.

\section{Resultados}

A primeira rodada de análise fatorial exploratória sugeriu dois fatores: o primeiro, composto por sete itens e o segundo, com três itens. Entretanto, nessa composição, dois itens carregaram simultaneamente nos dois fatores ("7 - Em comparação com outras pessoas, eu posso fazer a maioria das tarefas muito bem" e " 8 - Mesmo quando 
as coisas estão difíceis, eu consigo desempenhos muito bons"), tendo apenas o item " $4-\mathrm{Eu}$ acredito que posso ter sucesso, sem muito esforço, nas metas que estabeleço para mim mesmo", carregado unicamente no segundo fator. A composição do segundo fator, portanto, foi extremamente frágil, não justificando a sua permanência.

Seguindo a indicação do scree plot e da fundamentação teórica, forçou-se a extração de um fator único. Nessa segunda análise, embora os indicadores tenham sido positivos, o item 4 apresentou comunalidade muito abaixo dos demais, assim como carga fatorial baixa em comparação aos demais itens (.302). Diante disso, decidiu-se pela exclusão desse item. O resultado demonstrou a presença de um único fator formado por sete itens. Com base nos achados do Estudo 1 e com a análise da consistência interna da escala se itens fossem excluídos (a redução seria apenas de 0,02 no alfa de Cronbach), decidiu-se testar a solução sem o item 1, a fim de encontrar a versão da escala que pudesse se mostrar mais estável em diferentes amostras. Conforme previsto, essa foi a melhor solução encontrada em termos de indicadores da escala. Após os procedimentos citados, a nova solução encontrada (sem pré-determinar a quantidade de fatores) foi de uma solução de seis itens que, juntos, explicaram $54,73 \%$ da variação do construto, com KMO de 0,846.

Com base na solução encontrada pela AFE, conduziu-se a AFC, cujos resultados podem ser visualizados na Figura 2. Foi necessário inserir um parâmetro de ajuste entre os erros residuais dos itens 7 e 8 a fim de alcançar os indicadores ideais.

O modelo apresentou índices de ajuste satisfatórios $\left(\chi^{2} / g l=2,98 ; \mathrm{GFI}=0,97 ; \mathrm{CFI}=0,97 ; \mathrm{TLI}=0,94\right.$; RMSEA $=0,08)$, assim como níveis adequados de consistência interna da escala, sendo o $\alpha=0,830$.

Tabela 2

AFE da Escala Geral de Autoeficácia - Estudo 2

\begin{tabular}{lrr}
\hline \multicolumn{1}{c}{ Itens } & Componente & \multicolumn{1}{c}{$h^{2}$} \\
\cline { 2 - 3 } 5. Eu vou ser capaz de superar com êxito muitos desafios. & 0,793 & 0,628 \\
8. Mesmo quando as coisas estão difíceis, eu consigo desempenhos muito bons. & 0,776 & 0,602 \\
2. Quando me deparo com tarefas difíceis, tenho certeza de que vou realizá-las. & 0,748 & 0,560 \\
3. Em geral, eu acho que posso obter os resultados que são importantes para mim. & 0,727 \\
6. Eu acredito que posso realizar de forma eficaz muitas tarefas diferentes. & 0,528 \\
7. Em comparação com outras pessoas, eu posso fazer a maioria das tarefas muito bem. & 0,725 \\
& 0,526 \\
Valor próprio & 0,664 \\
Número de itens & 3,28 \\
\% da variância explicada & 6 \\
Alfa de Cronbach & 54,73 \\
\hline
\end{tabular}

Legenda. F1=Autoeficácia; $h^{2}=$ Comunalidade. Fonte. Dados da Pesquisa

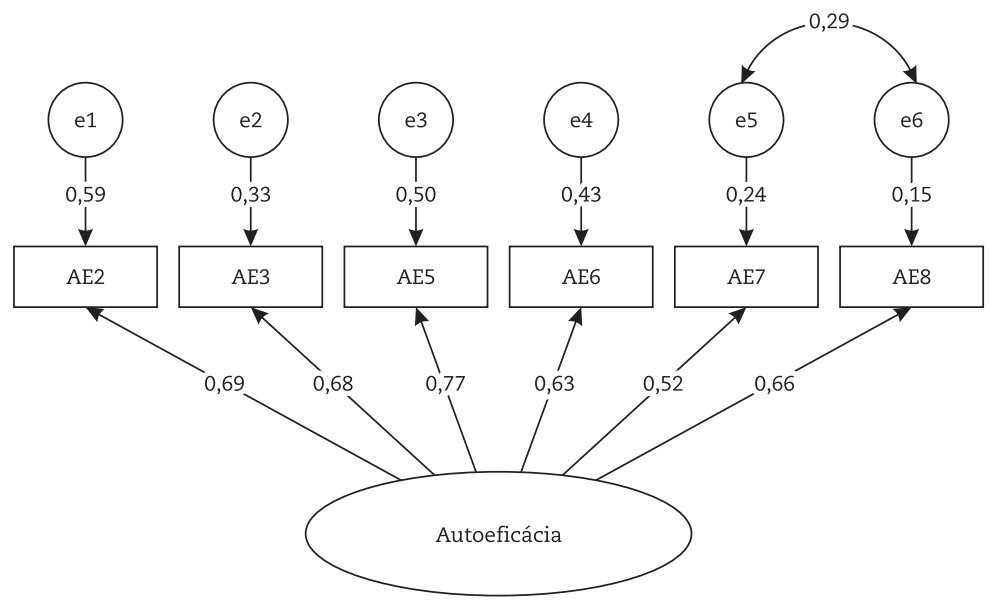

Figura 2. Estrutura do modelo da Escala Geral de Autoeficácia a partir dos dados do Estudo 2 


\section{Discussão Geral}

A presente pesquisa realizou, pela primeira vez, a validação da NGSE para o Brasil. A análise da escala demonstrou que o instrumento permite avaliar de forma estável a autoeficácia. No que tange à importância dessa pesquisa, cumpre ressaltar que o instrumento aqui proposto reteve somente seis itens dos oito originais de Chen et al. (2001). Dessa forma, apresenta-se uma composição de itens diferente daquela comumente utilizada.

A realização dos dois estudos possibilitou um aumento da generalização da escala, uma vez que a "nova escala" do Estudo 1 foi administrada a outra amostra independente no Estudo 2. Seguindo as recomendações de Hinkin (1998), a replicação no Estudo 2 incluiu análise fatorial exploratória, confirmatória, avaliação da confiabilidade, validade e consistência interna.

Os resultados de ambos os estudos demonstram qualidades psicométricas bastante satisfatórias. Ao se comparar os resultados de validação do estudo $01(\alpha=0,785$, valor próprio de 2,96 , variância explicada $49,37 \%$ ) com os obtidos na validação da escala por Chen et al. (2001) $(\alpha=0,870$, valor próprio $=4,17,52 \%$ da variância explicada), percebe-se que os indicadores do primeiro estudo nacional ficaram apenas levemente abaixo dos valores obtidos no estudo original. Quando os dados originais são comparados com resultados os obtidos no Estudo 02 ( $\alpha=0,830$, valor próprio de 3,28, variância explicada 54,73\%), identifica-se, mais uma vez, que os valores encontrados são próximos aos de Chen et al. (2001), tendo, inclusive, a variância explicada sido maior na validação nacional da escala. Nesse sentido, a escala aqui validada apresenta bom índice de consistência interna, assim como bons índices de ajuste do modelo, mesmo com menos itens. Tal fato, torna a NGSE uma opção viável para a mensuração da autoeficácia no contexto brasileiro.

Além de uma ferramenta de pesquisa acadêmica, o instrumento pode ser de grande valia nos estudos direcionados a contextos práticos, como, por exemplo, o organizacional e o educacional. Salienta-se que, por essa escala ser uma medida geral de autoeficácia, ela pode ser usada em variadas áreas, possibilitando comparações de desempenho em diferentes situações e cotidianos. Permite conhecer a confiança do indivíduo em sua capacidade de atingir objetivos, possibilitando a introdução de estratégias para o cuidado e promoção de motivação para a consecução de determinadas tarefas e enfrentamento de dificuldades, seja por intermédio de modificações em suas crenças, experiências de sucesso, experiências vicariantes ou modelagem.

Somado a isso, para além da sua importância já corroborada, a autoeficácia ainda apresenta um potencial de aplicação a ser investigado. Por fim, considera-se importante que a escala aqui apresentada continue a ser testada quanto à sua confiabilidade e validade em diferentes contextos socioculturais da realidade brasileira.

\section{Agradecimentos.}

Não há menções.

\section{Financiamento}

A presente pesquisa não recebeu nenhuma fonte de financiamento sendo custeada com recursos dos próprios autores.

\section{Contribuições dos autores}

Declaramos que todos os autores participaram da elaboração do manuscrito. Especificamente, o(s) autor(es) Laércio André Gassen Balsan, Laila Leite Carneiro, Antonio Virgílio Bittencourt Bastos e Vânia Medianeira Flores Costa participou(ram) da redação inicial do estudo - conceitualização, investigação, visualização, o(s) autor(es) Laércio André Gassen Balsan e Laila Leite Carneiro participou(ram) da análise dos dados, e o(s) autor(es) Laércio André Gassen Balsan, Laila Leite Carneiro, Antonio Virgílio Bittencourt Bastos e Vânia Medianeira Flores Costa participou(ram) da redação final do trabalho - revisão e edição.

\section{Disponibilidade dos dados e materiais}

Todos os dados e sintaxes gerados e analisados durante esta pesquisa serão tratados com total sigilo devido às exigências do Comitê de Ética em Pesquisa em Seres Humanos. Porém, o conjunto de dados e sintaxes que apoiam as conclusões deste artigo estão disponíveis mediante razoável solicitação ao autor principal do estudo.

\section{Conflito de interesses}

Os autores declaram que não há conflitos de interesses.

\section{Referências}

Araújo, M., \& Moura, O. (2011). Estrutura factorial da General Self-Efficacy Scale (Escala de Auto-Eficácia Geral) numa amostra de professores portugueses. Laboratório de Psicologia, 9(1), 95-105. doi: 10.14417/lp.638

Azzi, R. G., \& Polydoro, S. (2006). Autoeficácia proposta por Albert Bandura. Em R. G. Azzi \& S. A. J. Polydoro (Eds.). Autoeficácia em diferentes contextos (pp. 9-23). Campinas: Alínea.

Bandura, A. (1977) Self-efficacy: Toward a unifying theory of behavioral change. Psychological Review, 84(2), 191-215. doi: 10.1037/0033295X.84.2.191 
Bandura, A. (1982). Self-efficacy mechanism in human agency. American Psychologist, 37(2), 122-147. doi: 10.1037/0003-066X.37.2.122

Bandura, A. (2001). Social cognitive theory: An agentic perspective. Annual Review of Psychology, 52(1), 1-26. doi: 10.1146/annurev.psych.52.1.1

Bandura, A. (2002). Social cognitive theory in cultural context. Journal of Applied Psychology: An International Review, 51(2), 269-290. doi: 10.1111/1464-0597.00092

Bandura, A. (2008). A evolução da teoria social cognitiva. Em A. Bandura, R. G. Azzi, S. A. J. Polydoro (Eds.). Teoria social cognitiva: Conceitos básicos (pp. 15-41). Porto Alegre: Artmed.

Bandura, A., \& Locke, E. A. (2003). Negative Self-Efficacy and Goal Effects Revisited. Journal of Applied Psychology, 88(1), 87-99. doi: 10.1037/0021-9010.88.1.87

Bandura, A., Taylor, C. B., Williams, S. L., Mefford, I. N., \& Barchas, J. D. (1985). Catecholamine secretion as a function of perceived coping self-efficacy. Journal of Consulting and Clinical Psychology, 53(3), 406-414.

Barros, M., \& Batista-dos-Santos, A. C. (2010). Por dentro da autoeficácia: Um estudo sobre seus fundamentos teóricos, suas fontes e conceitos correlatos. Revista Espaço Acadêmico, 10(112), 1-9.

Byrne, B. M. (2016). Structural equation modeling with Amos: Basic concepts, applications, and programming. Londres: Lawrence Erlbaum.

Bzuneck, J. A., \& Guimarães, S. E. R. (2003). Crenças de eficácia de professores: Validação da escala de Woolfolk e Hoy. Revista Psico-USF, $8(2), 137-143$.

Canrinus, E. T., Helms-Lorenz, M., Beijaard, D., Buitink, J., \& Hofman, A. (2012). Self-efficacy, job satisfaction, motivation and commitment: Exploring the relationships between indicators of teachers' professional identity. European Journal of Psychology of Education, 27(1), 115132. doi: 10.1007/s10212-011-0069-2

Caprara, G. V., Barbaranelli, C., Steca, P., \& Malone, P. S. (2006). Teachers' self-efficacy beliefs as determinants of job satisfaction and students' academic achievement: A study at the school level. Journal of School Psychology, 44(6), 473-490. doi: 10.1016/j.jsp.2006.09.001

Cardoso, H. F., \& Baptista, M. N. (2019). Self-Efficacy Scale at Work (EAE-T): Construction and Psychometric Studies. Gerais: Revista Interinstitucional de Psicologia, 12(1), 3-18. doi: 10.36298/gerais2019120102

Chan, W.Y., Lau, S., Nie, Y., Lim, S., \& Hogan, D. (2008). Organizational and personal predictors of teacher commitment: The mediating role of teacher efficacy and identification with school. American Educational Research Journal, 45(3), 597-630. doi: 10.3102/0002831208318259

Chen, G., Gully, S. M., \& Eden, D. (2001). Validation of a new general self-efficacy scale. Organizational Research Methods, 4(1), 62-83. doi: $10.1177 / 109442810141004$

Chen, G., Gully, S. M., Whiteman, J. A., \& Kilcullen, B. N. (2000). Examination of relation-ships among trait-like individual differences, state-like individual differences, and learning performance. Journal of Applied Psychology, 85(6), 835-847. doi: 10.1037/0021-9010.85.6.835

Ciani, K. D., Summers, J. J., \& Easter, M. A. (2008). A “top-down" analysis of high school teacher motivation. Contemporary Educational Psychology, 33(4), 533-560. doi: 10.1016/j.cedpsych.2007.04.002

Cruz, A. C., Angelo, M., \& Santos, B. P. (2017). Self-efficacy scale for the establishment of good relationships with families in neonatal and pediatric hospital settings. Revista da Escola de Enfermagem da USP, 51, 1-8. doi: 10.1590/s1980-220x2016033803222

Fida, R., Laschinger, H. K. S., \& Leiter, M. P. (2016). The protective role of self-efficacy against workplace incivility and burnout in nursing: A time-lagged study. Health Care Management Review, 43(1), 21-29. doi: 10.1097/HMR.0000000000000126

Field, A. (2009). Descobrindo a Estatística Usando o SPSS-5. Penso Editora.

Gist, M. (1987). Self-efficacy: Implications for organizational behavior and human resource management. Academy of Management Review, 12(3), 472- 485. doi: 10.5465/amr.1987.4306562

Guillemin, F., Bombardier, C., \& Beaton, D. (1993). Cross-cultural adaptation of health-related quality of life measures: Literature review and proposed guidelines. Journal of Clinical Epidemiolgy, 46(12), 1417-1432. doi: 10.1016/0895-4356(93)90142-N

Hair, J. f., Black, W. C. B., Babin, B. J., Anderson, R. E., \& Tatham, R. L. (2009). Análise multivariada de dados. Porto Alegre: Bookman.

Hinkin, T. R. (1998). A Brief Tutorial on the Development of Measures for Use in Survey Questionnaires [Electronic version]. Recuperado de Cornell University, School of Hotel Administration http://scholarship.sha.cornell.edu/articles/521

Iaochite, T. R., \& Azzi, R. G. (2012). Escala de fontes de autoeficácia docente: Estudo exploratório com professores de Educação Física. Psicologia Argumento, 30(71), 659-669. doi: 10.7213/psicol.argum.7472

Judge, T. A., Bono, J. E., Erez, A., \& Locke, E. A. (2005). Core self-evaluations and job and life satisfaction: The role of self-concordance and goal attainment. Journal of Applied Psychology, 90(2), 257-268. doi: 10.1037/0021-9010.90.2.257

Judge, T. A., Erez, A., \& Bono, J. A. (1998). The power of being positive: The relation between positive self-concept and job performance. Human Performance, 11(2), 167-187. doi: 10.1080/08959285.1998.9668030

Kusumaninggati, G., Mukhtar, M., \& Sujanto B. (2018). The influence of self-efficacy, job satisfaction and organizational commitment toward Organizational Citizenship Behavior (Ocb) of Teachers of Private Vocational Schools in South Jakarta. International Journal of Scientific Research and Management, 6(6), 406-414. doi: 10.18535/ijsrm/v6i6.el06

Latham, G. P., \& Pinder, C. P. (2005). Work motivation theory and research at the dawn of the twenty-first century. Annual Review of Psychology, 56, 485-516. doi: 10.1146/annurev.psych.55.090902.142105

Lee, K., \& Kim, Y. (2019). Effects of self-efficacy and job stress on organizational commitment among clinical dental hygienists. Journal of Dental Hygiene Science, 19(1), 60-66. doi: 10.17135/jdhs.2019.19.1.60

Lee, J., \& Oh, S. (2015). Effects of positive affect and negative affect on the life satisfaction: The role of work self-efficacy and work meaningfulness. Journal of Korea Society of Computer and Information, 20(2), 187-195. doi: 10.9708/jksci.2015.20.2.187

Lent, R. W., Brown, S. D., \& Hackett, G. (1994). Toward a unifying social cognitive theory of career and academic interest, choice, and performance. Journal of Vocational Behavior, 45(1), 79-122. doi: 10.1006/jvbe.1994.1027

Lloyd, J., Bond, F. W., \& Flaxman, P. E. (2017). Work-Related Self-Efficacy as a Moderator of the Impact of a Worksite Stress Management Training Intervention: Intrinsic Work Motivation as a Higher Order Condition of Effect. Journal of Occupational Health Psychology, 22(1), 115-127. doi: 10.1037/ocp0000026 
Lubbers, R., Loughlin, C., \& Zweig, D. (2005). Young workers' job self-efficacy and affect: Pathways to health and performance. Journal of Vocational Behavior, 67(2), 199-214. doi: 10.1016/j.jvb.2004.03.002

Luszczynska, A., Scholz, U., \& Schwarzer, R. (2005). The General Self- Efficacy Scale: Multicultural validation studies. The Journal of Psychology, 139(50), 439-457. doi: 10.3200/JRLP.139.5.439-457

Malhotra, N. F. N. (2019). Pesquisa de marketing: Uma orientação aplicada. Porto Alegre: Bookman.

Olaz, F. (2003). Modelo social cognitivo del desarrollo de carrera. Revista Evaluar, 3(1), 5-34. doi: 10.35670/1667-4545.v3.n1.605

Oriá, M. O. B, \& Ximenes, L. B. (2010). Tradução e adaptação cultural da Breastfeeding Self-Efficacy Scale para o português. Acta Paulista de Enfermagem, 23(2), 230-238. doi: 10.1590/S0103-21002010000200013

Pajares, F. (1997). Current directions in self-efficacy research. Em M. L. Maehr \& P. H. Pintrich (Eds.) Advances in motivation and achievement(pp. 1-49). Greenwich: JAI Press.

Pajares, F., \& Olaz, F. (2008). Teoria social cognitiva e autoeficácia: Uma visão geral. Em A. Bandura, R. G. Azzi \& S. Polydoro (Eds.). Teoria social cognitiva: Conceitos básicos (pp. 97-114). Porto Alegre: Artmed.

Perdue, S. V., Reardon, R. C., \& Peterson, G. W. (2007). Person-environment congruence, self-efficacy and environmental identity in relation to job satisfaction: A career decision theory perspective. Journal of Employment Counseling, 44(1), 29-39. doi: 10.1002/j.21611920.2007.tb00022.x

Perera, H. N., Granziera, H., \& Mcllveen, P. (2018). Profiles of teacher personality and relations with teacher self-efficacy, work engagement, and job satisfaction. Personality and Individual Differences, 120, 171-178. doi: 10.1016/j.paid.2017.08.034

Reizer, A., \& Hetsroni, A. (2015). Caregiving representations at work and the moderating role of job self-efficacy. Psychological Reports: Employment Psychology \& Marketing, 116(1), 60-73. doi: 10.2466/01.PR0.116k12w2

Rhee, S., Hur, W., \& Kim, M. (2017). The relationship of coworker incivility to job performance and the moderating role of self-efficacy and compassion at work: The Job Demands-Resources (JDR) Approach. Journal of Business and Psychology, 32(1), 711-726. doi: 10.1007/ s10869-016-9469-2

Rots, I., Aelterman, A., Vlerick, P., \& Vermeulen, K. (2007). Teacher education, graduates' teaching commitment and entrance into the teaching profession. Teaching and Teacher Education, 23(5), 543-556. doi: 10.1016/j.tate.2007.01.012

Salas, E., \& Cannon-Bowers, J. (2001). The science of training: A decade of progress. Annual Review of Psychology, 52(1), 471-499. doi 10.1146/annurev.psych.52.1.471

Sbicigo, J. B., Teixeira, M. A. P., Dias, A. C. G., \& Dell'aglio, D. D. (2012). Propriedades psicométricas da escala de autoeficácia geral percebida (EAGP). Psico, 43(2), 139-146.

Schepers, C., De Gieter, S., Pepermans, R., Du Bois, C., Caers, R., \& Jegers, M. (2005). How are employees of the nonprofit sector motivated? a research need. Nonprofit Management \& Leadership, 16(2), 191-208. doi: 10.1002/nml.100

Scherbaum, C. A., Cohen-Charash, Y., \& Kern, M. J. (2006). Measuring general self-efficacy: A comparison of three measures using item response theory. Educational and Psychological Measurement, 66(6), 1047-1063. doi: 10.1177/0013164406288171

Scholz, U., Doña, B. G., Sud, S., \& Schwarzer, R. (2002). Is general self-efficacy a universal construct? psychometric findings from 25 countries. European Journal of Psychological Assessment, 18(3), 242-251. doi: 10.1027/1015-5759.18.3.242

Schwarzer, R. (1992). Self-efficacy: Thought control of action. Washington: Hemisphere, 1992.

Schwarzer, R., \& Hallum, S. (2008). Perceived teacher self-efficacy as a predictor of job stress and burnout: Mediation analyses. Applied Psychology, 57(2), 152-171. doi: 10.1111/j.1464-0597.2008.00359.x

Schwarzer, R., \& Jerusalem, M. (1995). Generalized Self-Efficacy Scale. Em J. Weinman, S. Wright \& M. Johnston. (Eds.), Measures in health psychology: A user's portfolio. Causal and control beliefs (pp. 35-37). Windsor: Nfer-Nelson.

Schwarzer, R. \& Schmitz, G. S. (2004). Perceived Self-efficacy as a resource factor in teachers. Em M. Salanova, R. Grau, I. M. Martínez, E. Cifre, S. Llores, \& M. Garcíarenedo (Eds.). Nuevos Horizontes en la Investigación sobre la Autoeficacia (pp. 229-236). Castelló de la Plana: Publicacions de la Universitat Jaume I.

Sherer, M., \& Adams, C. H. (1983). Construct validation on the self-efficacy scale. Psychological Reports, 53(3), 899-902. doi: 10.2466/ pr0.1983.53.3.899

Sherer, M., Maddux, J. E., Mercandante, B., Prentice-Dunn, S., Jacobs, B., \& Rogers, R. W. (1982). The Self-Efficacy Scale: Construction and validation. Psychological Reports, 51(2), 663-671. doi: 10.2466/pr0.1982.51.2.663

Smeds, M. R., Janko, M. R., Allen, S., Amanhwah, K., Arnell, T., Ansari, P. .. \& Yoo, P. (2019). Burnout and its Relationship with Perceived Stress, Self-Efficacy, Depression, Social Support, and Programmatic Factors in General Surgery Residents. The American Journal of Surgery, 219 (6), 907-912. doi: 10.1016/j.amjsurg.2019.07.004

Snyder, C. R., \& Lopez, S. J. (2009). Psicologia Positiva: Uma abordagem científica e prática das qualidades humanas. Porto Alegre: Artmed.

Souza, I., \& Souza M. A. (2004). Validação da Escala de Autoeficácia Geral Percebida. Revista Universidade Rural, 26(1), 12-17.

Stajkovic, A. D., \& Luthans, F. (1998). Self-efficacy and work-related task performance: A meta-analysis. Psychological Bulletin, 124(2), 240261. doi: $10.1037 / 0033-2909.124 .2 .240$

Tabachnick, B. G., \& Fidell, L. S. (2019). Using multivariate statistics. California: Pearson.

Tsai, M., Tsai, C., \& Wang, Y. (2011). A study on the relationship between leadership style, emotional intelligence, self-efficacy and organizational commitment: A case study of the Banking Industry in Taiwan. African Journal of Business Management, 5(13), $5319-5329$. doi: 10.5897/AJBM10.932

Wood, R. E., \& Bandura, A. (1989). Social cognitive theory of organizational management. Academy of Management Review, 14(3), 361-384. doi: 10.5465/amr.1989.4279067

Yousaf, A., \& Sanders, K. (2012). The role of job satisfaction and self-efficacy as mediating mechanisms in the employability and affective organizational commitment relationship: A case from a Pakistani University. Thunderbird International Business Review, 54(6), 907-919. doi: $10.1002 /$ tie. 21511 
Zimmerman, B. J. (2000). Self-efficacy: An essential motive to learn. Contemporary Educational Psychology, 25(1), 82-91. doi: 10.1006/ ceps.1999.1016

\section{Sobre os autores}

Laércio André Gassen Balsan é Doutor em Administração. Atua como pesquisador nos seguintes temas: Comportamento Organizacional, Gestão de Pessoas, Gestão Pública, Gestão Universitária, Qualidade de vida e Estresse.

Laila Leite Carneiro é Doutora em Psicologia. Professora Adjunta do Instituto de Psicologia da Universidade Federal da Bahia. Pesquisa temas relacionados à aplicação da Psicologia Positiva no campo das organizações e do trabalho.

Antonio Virgílio Bittencourt Bastos é Doutor em Psicologia. Professor titular de Psicologia Social das Organizações, no Instituto de Psicologia da Universidade Federal da Bahia, Pesquisador $1^{\text {a }}$ do CNPq.

Vânia Medianeira Flores Costa é Doutora em Administração, Professora Associada do Departamento de Ciências Administrativas da Universidade Federal de Santa Maria.

\section{Como citar este artigo}

Balsan et al. (2020). Adaptação e validação da Nova Escala Geral de Autoeficácia. Avaliação Psicológica, 19(4), 409-419. http://dx.doi. org/10.15689/ap.2020.1904.16654.07 